\title{
Cryptochromes modulate E2F family transcription factors
}

\begin{abstract}
Alanna B. Chan ${ }^{1}$, Anne-Laure Huber ${ }^{1,2} \&$ Katja A. Lamia ${ }^{1 *}$
Early 2 factor (E2F) family transcription factors participate in myriad cell biological processes including: the cell cycle, DNA repair, apoptosis, development, differentiation, and metabolism. Circadian rhythms influence many of these phenomena. Here we find that a mammalian circadian rhythm component, Cryptochrome 2 (CRY2), regulates E2F family members. Furthermore, CRY1 and CRY2 cooperate with the E3 ligase complex SKP-CULLIN-FBXL3 $\left(\mathrm{SCF}^{\mathrm{FBX}}{ }^{3}\right)$ to reduce E2F steady state protein levels. These findings reveal an unrecognized molecular connection between circadian clocks and cell cycle regulation and highlight another mechanism to maintain appropriate E2F protein levels for proper cell growth.
\end{abstract}

\section{Circadian rhythms and the cell cycle}

Circadian rhythms allow organisms to anticipate daily environmental changes in accordance with the Earth's rotation. In mammals, nearly every cell has a circadian clock $^{1}$. Mammalian circadian rhythms are generated by a core molecular clock composed of a transcription translation feedback loop (TTFL); the heterodimer circadian locomotor output cycles kaput (CLOCK) and Brain and muscle ARNT-like protein 1 (BMAL1) drives transcription of their own repressors Period (PER 1-3) and Cryptochrome (CRY1-2). Circadian clocks have been found to influence cell cycle progression, possibly through transcriptional regulation of $c-M y c, C c n d 1$, and/or Wee- $1^{2,3}$. It is becoming increasingly clear that the circadian clock and the cell cycle can interact dynamically, but the molecular mechanisms connecting these two biological oscillators are largely unknown ${ }^{2,4,5}$.

TTFL components have been postulated to have cellular functions outside circadian rhythm generation ${ }^{2,6,7}$. CRY1/2 bind many thousands of unique sites in the genome independent of CLOCK and BMAL1 ${ }^{8}$, and interact with several non-circadian transcription factors including nuclear hormone receptors ${ }^{9-12}$ and c-MYC ${ }^{13}$. In addition, CLOCK, BMAL1, PERs, and CRYs have been implicated in cell cycle control. CLOCK and BMAL1 overexpression in a human colon cancer cell line prevented entry into $S$ phase possibly through decreased CYCLIN $\mathrm{D} 1$ protein levels ${ }^{14}$. In wildtype mice subjected to partial hepatectomy (PH), hepatocytes entered $\mathrm{M}$ phase faster when the surgery was performed in the afternoon compared to those that underwent $\mathrm{PH}$ at night, possibly due to lower Weel expression in the afternoon, suggesting a link between time of day and cell cycle progression ${ }^{3}$. PER has been implicated in cell cycle control via p16-Ink4A, which is rhythmically expressed throughout the day and is suppressed by PER $2^{6}$. PER2 also modulates the stability of P53 $3^{15,16}$, which could influence the cell cycle in unstressed conditions and help the cell anticipate genotoxic stress. Another important protein involved in cell cycle control is c-MYC - a widely known proto-oncogene. We recently discovered that CRY2 acts as a co-factor for the SKP-CULLIN-FBXL3 ( $\mathrm{SCF}^{\mathrm{FBXL} 3}$ ) complex to promote degradation of phosphorylated substrates, including $\mathrm{c}-\mathrm{MYC}^{13}$ and Tousled-like kinase 2 (TLK2) ${ }^{17}$.

\section{The E2F family}

The Early 2 Factors (E2Fs) are a family of eight winged-helix transcription factors that are key to regulating cell cycle progression from G1 to S phase among other functions ${ }^{18,19}$. E2F1, E2F2, and E2F3a are expressed in early G1 through $S$ phase and are believed to promote $S$ phase initiation by activating genes involved in the G1 to $S$ phase transition ${ }^{18}$. E2F3b, E2F4, E2F6, E2F5, E2F7, and E2F8 act as repressors and are expressed later in the cell cycle $^{18}$. Since E2Fs are crucial in mediating cell cycle progression their protein levels must by tightly regulated. $\mathrm{SCF}^{\mathrm{SKP} 2}$ interacts with the N-terminus of $\mathrm{E} 2 \mathrm{~F} 1$ and promotes ubiquitination and degradation of E2 $\mathrm{F}^{20}$. The anaphase-promoting complex (APC) promotes E2F1 degradation during $\mathrm{M}$ phase and this requires interaction between the $\mathrm{C}$-terminus of $\mathrm{E} 2 \mathrm{~F} 1$ and the $\mathrm{APC}$ adaptor protein $\mathrm{CDH} 1^{21}$. $\mathrm{APC} / \mathrm{C}^{\mathrm{CDH}} 1$ also stimulates degradation of E2F3, E2F7, and E2F8 ${ }^{22,23}$. Recently, CYCLIN F has been shown to promote ubiquitination of E2F1-3a ${ }^{24}$. Here, we demonstrate that $\mathrm{CRY} 1 / 2$ and $\mathrm{SCF}^{\mathrm{FBXL} 3}$ reduce the steady state protein levels of some E2F family members.

${ }^{1}$ Department of Molecular Medicine, The Scripps Research Institute, 10550 North Torrey Pines Road, La Jolla, CA, 92037, USA. ${ }^{2}$ Centre de Recherche en Cancerologie de Lyon, 28 rue Laennec, 69008, Lyon, France. *email: klamia@ scripps.edu 
A

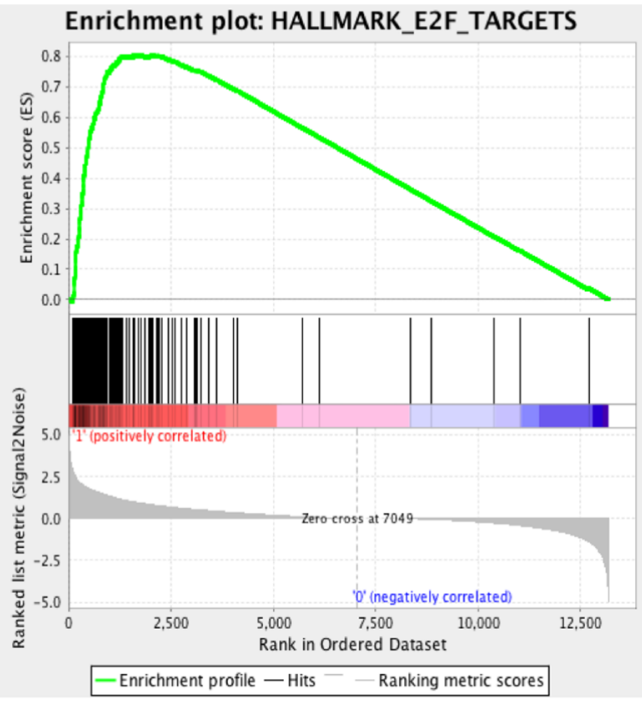

C

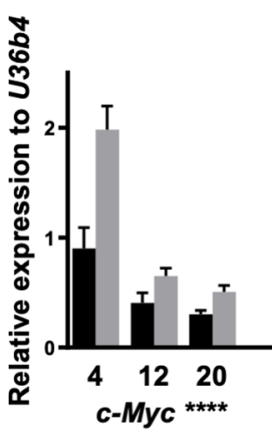

B

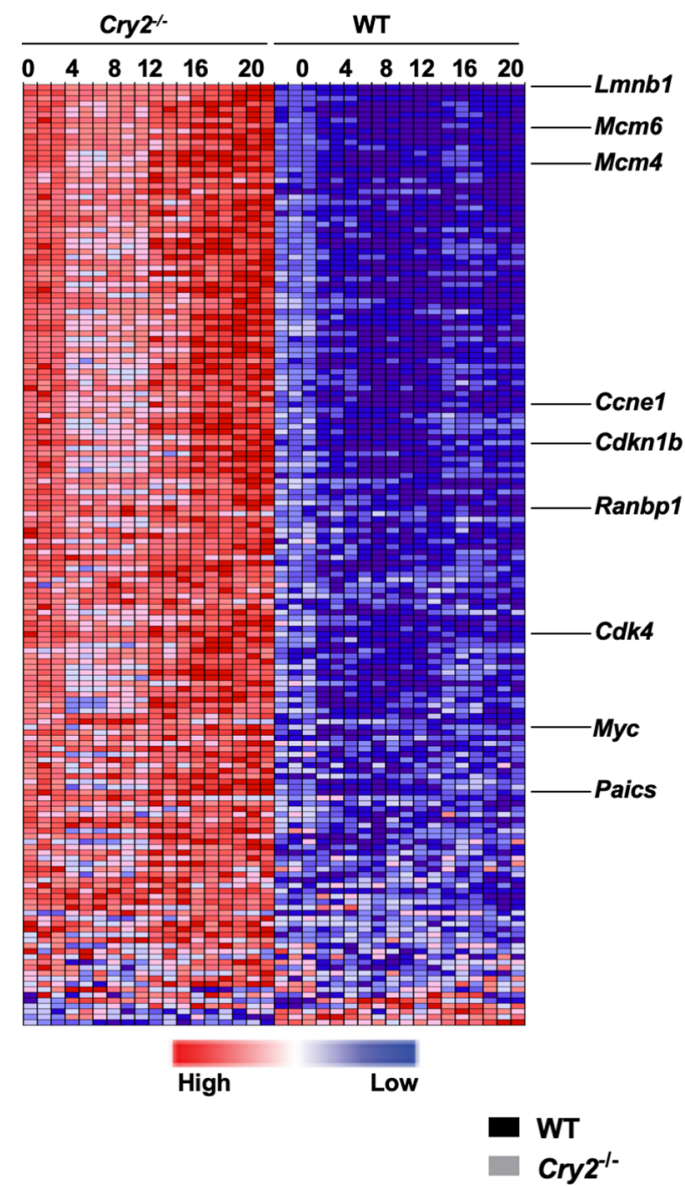

Figure 1. E2F target genes are upregulated in the absence of CRY2. (A) GSEA enrichment plot: The top portion shows the running enrichment score (ES) for the gene set as the analysis walks down the ranked list. The middle portion shows where the members of the gene set appear in the ranked list of genes. The bottom portion shows the value of the ranking metric as you move down the list of ranked genes. The ranking metric measures a gene's correlation with a phenotype (FDR q-value $=0.07)$. RNA sequencing data from ${ }^{13}$. $(\mathbf{B})$ Heatmap from RNA sequencing in primary MEFs at the indicated times after circadian synchronization with dexamethasone. Colors represent high (red) to blue (low) expression. The associated ranked gene names are provided in Table S1. (C) Expression of indicated transcripts in primary WT (black) and $\mathrm{Cr}_{2} 2^{-I-}$ (gray) MEFs at indicated times (hours) after dexamethasone treatment. Data represent mean \pm s.e.m. of 2-3 biological triplicates each analyzed in triplicate. ${ }^{*} \mathrm{p}<0.05,{ }^{*} \mathrm{p}<0.01,{ }^{* * * *} \mathrm{p}<0.0001$ by two-way ANOVA with Tukey's multiple comparisons for effect of genotype.

These findings highlight a more widespread substrate repertoire of $\mathrm{CRY} 2$ and $\mathrm{SCF}^{\mathrm{FBXL} 3}$ mediated degradation and further supports the interconnection between circadian clocks and cell cycle progression.

\section{Results}

E2F target genes are upregulated in $\mathrm{Cry2}^{-1-}$ compared to Wildtype MEFs. To determine how the global transcriptome is altered in wildtype (WT) and $\mathrm{Cry} 2^{-/-}$cells in an unbiased manner, we performed gene set enrichment analysis (GSEA) on RNA-sequencing (RNA-seq) data from WT and Cry $2^{-1-}$ mouse embryonic fibroblasts (MEFs). Following synchronization of circadian rhythms with the synthetic glucocorticoid agonist dexamethasone ${ }^{13}$. When analyzed as a group ${ }^{25}$, expression of 200 transcripts defined as hallmark ${ }^{26} \mathrm{E} 2 \mathrm{~F}$ target genes (Table S1) exhibits a small but highly consistent elevation in Cry $2^{-1-}$ MEFs compared to WT (Fig. 1A,B). We used RNA samples isolated from independently synchronized WT and $\mathrm{Cry}^{-1-}$ cells to evaluate the reproducibility of 
this finding by directly measuring the expression of a subset of E2F target genes (Figs. 1C and Fig. S1). The expression of E2f family members themselves was largely unchanged in Cry $2^{-I-} \mathrm{MEFs}$, though E2f2 and E2f7 transcripts tend to be increased late and decreased early in the circadian cycle, respectively (Fig. S1). These data suggest that upregulation of E2F target genes involves a post-transcriptional mechanism.

E2F family members interact with CRY1, CRY2, and FBXL3. As we previously found that CRY2 can act as a co-factor for SCF ${ }^{\mathrm{FBXL} 3}$ to stimulate ubiquitination and degradation of C-MYC and TLK2 ${ }^{13,17}$, we hypothesized that CRY2 could act as a co-factor for E2F family member degradation in a similar manner. We assessed interaction between E2F family members and $\mathrm{SCF}^{\mathrm{FBXL} 3}$ combined with CRY1 or CRY2 using co-immunoprecipitation (co-IP). We investigated E2F1, E2F4, and E2F8 as representatives of the three major E2F subfamilies of activators and repressors (Fig. 2A). Indeed, we found that each of these FLAG-tagged E2F family members, when immunoprecipitated, can interact with FBXL3 and those interactions are enhanced in the presence of CRY1 and CRY2 to varying degrees (Fig. 2B,C). Both E2F1 and E2F8 preferentially interact with CRY2 compared to CRY1, while E2F4 interacts with CRY1 and CRY2. Conversely, when FLAG-tagged FBXL3 is used as bait for immunoprecipitation, its interactions with E2F1, E2F4, or E2F8 are increased in the presence of CRY1 and/or CRY2. E2F4 and E2F8 consistently interact to a greater degree with FBXL3 than does E2F1 (Fig. 2D,E).

Steady state levels of E2F1, E2F4, and E2F8 are decreased in the presence of CRY2 and FBXL3. CRY2 can act as a co-factor to enhance turnover of c-MYC and TLK2 through interaction with FBXL $3^{13,17}$. To determine whether CRY2 and FBXL3 similarly influence E2F family proteins, we measured overexpressed E2F protein levels in the presence or absence of overexpressed human CRY2 (hCRY2.1) and FBXL3. The steady state protein levels of all three E2F proteins studied (E2F1, E2F4, and E2F8) were markedly decreased in the presence of CRY2 and FBXL3 (Fig. 3A-C). This decrease of E2F1, E2F4, and E2F8 protein levels in the presence of human CRY1/CRY2 and FBXL3 was partially mitigated with treatment of MG-132, a reversible proteasome inhibitor. The impact of MG-132 treatment seems to be greatest for E2F4 in the presence of FBXL3 combined with either CRY1 or CRY2 and for E2F8 in the presence of CRY1 and FBXL3 (Fig. S2). The robust effect on steady state protein levels makes it difficult to interpret effects on the turnover of overexpressed proteins, but the turnover of overexpressed E2F1 appears to increase in the presence of overexpressed FBXL3 and CRY2 (Fig. S3).

Deletion of endogenous Cry1/2 impacts E2F protein levels. To determine whether endogenous CRY1 and CRY2 impact E2F protein levels, we stably expressed tetracycline-inducible FLAG-tagged human E2F1, E2F4, or E2F8 in WT or Cry-deficient adult mouse ear fibroblasts (AMEFs) (Figs. 4 and Fig. S4). Since E2F proteins are involved in cell cycle regulation, we induced their expression at low and high plating densities (20\% and $100 \%)$ to capture both proliferating and growth-arrested conditions. E2F1 protein levels are elevated when Cry1 is deleted, but not when Cry2 or both Cry1 and Cry2 are deleted at both plating densities (Fig. 4A-D). Even though E2F1 protein abundance is significantly increased in the absence of Cry1 (Fig. 4B,D), the E2F1 protein levels are highly variable, and loss of Cryl also seems to impact expression of exogenous E2F1 mRNA (Fig. S5) making us less confident in the biological significance of the increased E2F1 in the Cry $1^{-1-}$ genotype. E2F4 protein is robustly increased in cells lacking either CRY1 or CRY2, regardless of confluency (Fig. 4E-H). E2F8 protein levels are dramatically increased in the absence of Cry1, regardless of cell confluency (Fig. 4I-L) suggesting that CRY1 may play a more important role than CRY2 in the regulation of E2F8 protein abundance. We tried to detect endogenous mouse E2F1, E2F4, and E2F8 protein levels in AMEFs; however, we could not confidently detect endogenous mouse E2F1, E2F4, or E2F8. Although we were not able to confirm its specificity with shRNA or E2F8 ${ }^{-I-}$ cells, the anti-E2F8 antibody detects a protein at the correct molecular weight (indicated by arrow), which is increased in all Cry $1 / 2$-deficient genotypes, further supporting our findings regarding E2F8 (Fig. S6). Cry1/2 genotype had little to no effect on the expression of endogenous E2f1 or E2f4 mRNA (Fig. S5). Interestingly, as we have observed for other doxycycline-inducible systems ${ }^{17}$, doxycycline-induced expression of human E2F1 or E2F4 mRNA was sometimes elevated in Cry-deficient cells compared to WT (Fig. S5). Because the upregulation of doxycycline-induced expression of human E2F4 mRNA across the genotypes did not follow the same trends observed at the protein level (Fig. 4), CRYs seem to impact E2F4 protein abundance post-translationally. The robust impact on E2F8 protein level suggests that it may also be a target of CRY1/2-dependent post-translational regulation. All in all, these data support our hypothesis that CRY1 or CRY2 can decrease $\mathrm{E} 2 \mathrm{~F} 4$ and $\mathrm{E} 2 \mathrm{~F} 8$ protein levels by recruiting them to $\mathrm{SCF}^{\mathrm{FBX} 3}$.

\section{Discussion}

We find that E2F target genes are slightly but consistently upregulated in Cry2 $2^{-1-}$ MEFs compared to matched WT control cells. Cry $2^{-l-}$ MEFs proliferate faster than WT MEFs ${ }^{13}$, which may be effected by higher expression of c-MYC as we have previously documented ${ }^{13}$ and could also be influenced by enhanced E2F activity. CRY2 could also influence cell growth through its partner PER2, which has been found to modulate the stability and nuclear translocation of $\mathrm{p} 53^{15,27}$, and/or through the actions of the core clock activating complex of CLOCK and BMAL1. It is likely that CRY2 acts in multiple ways to influence cell growth; dissecting the relative contributions of each of these mechanisms to increased proliferation in CRY2-deficient cells will require extensive additional investigation.

Here, we find that both activator and repressor E2F family transcription factors interact with FBXL3 and these interactions are enhanced by CRY1 and/or CRY2. Intriguingly, the representative members of the repressor subfamilies, E2F4 and E2F8, interact more strongly with CRYs and FBXL3 than does the canonical activator E2F1. While we did not identify the region(s) of the E2F proteins that interact with FBXL3 and/or CRYs in this study, these differences likely reflect preferential interaction with amino acid sequences that are conserved in the repressor subfamilies. These observations are also consistent with the greater impact of CRY1/2 overexpression 


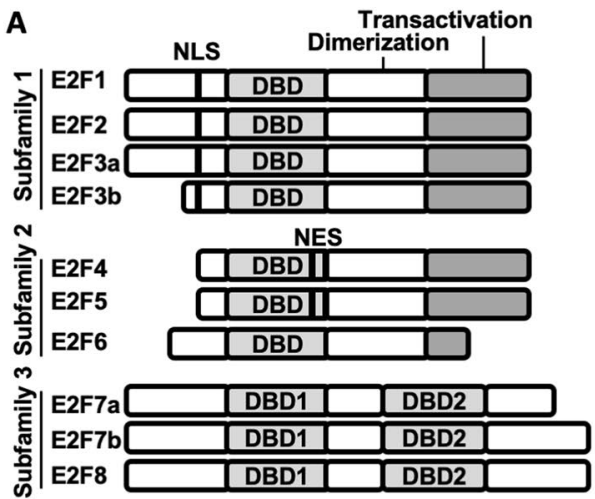

\section{B}

HA-mCRY1/2: 12 2 - 11122 2 - 11122 mFBXL3-V5: + + + + + + + + + + - FLAG-E2F4 FLAG-E2F1

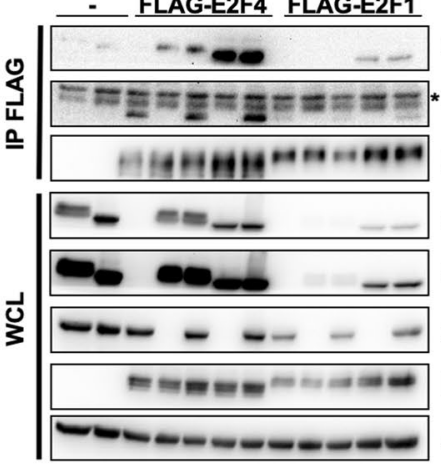

D

FLAG-mFBXL3

MYC-CRY1/2: - $121212-12$ - 12

HA-E2F4/1: $-\begin{array}{rllllllllll}4 & 4 & 4 & 1 & 1 & 4 & 4 & 4 & 1 & 1 & 1\end{array}$

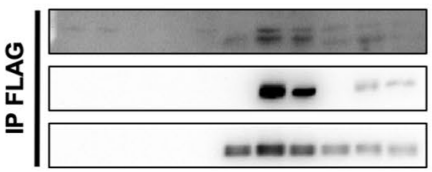

HA-E2F4/1

MYC-CRY1/2

FLAG-FBXL3

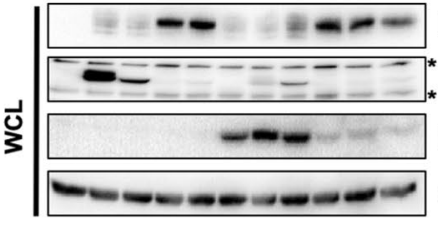

CRY1/2

*mFBXL3-V5

FLAG-E2F4/1

mFBXL3-V5

FLAG-E2F4/1

a-TUBULIN

CRY1/2 (short exp)

CRY1/2 (long exp)

E

C

HA-mCRY1/2: 12 - $111222-1122$

mFBXL3-V5: + + + + + + + + +

- FLAG-E2F4 FLAG-E2F8

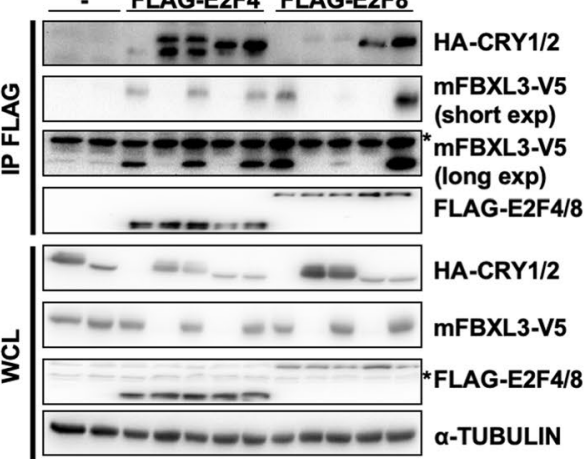

FLAG-mFBXL3

MYC-CRY1/2: - $121212-12$ - 12

HA-E2F4/8: $\quad-\begin{array}{llllllllll}4 & 4 & 8 & 8 & 4 & 4 & 4 & 8 & 8 & 8\end{array}$

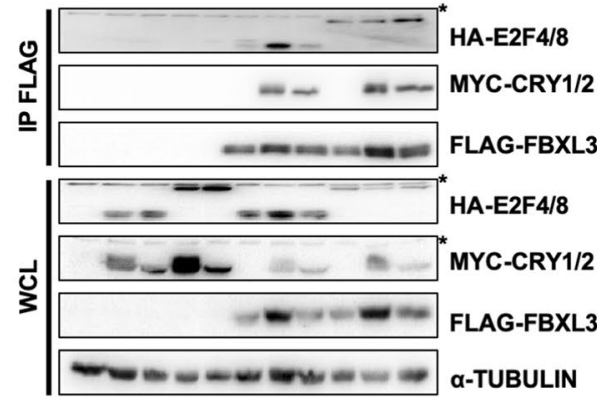

Figure 2. E2F1, E2F4, and E2F8 interact with CRYs and FBXL3. (A) Diagram of the three subfamilies of the E2F family, Nuclear localization signal, NLS; DNA binding domain, DBD; Nuclear export signal, NES. (B-E) Proteins detected by immunoblot (IB) following FLAG immunoprecipitation (IP) or in whole cell lysates (WCL) from HEK293T cells expressing the indicated plasmids with the indicated tags. (*) Denotes nonspecific band(s). Short-exp, short exposure; long exp, long exposure. Note: All uncropped blots are provided in Supplementary Information.

or genetic deletion on protein levels of overexpressed or endogenous repressor E2F family members, E2F4 and E2F8, compared to the activator E2F1.

The observed greater sensitivity of steady state protein levels of repressor E2F family members to the loss of Cry 2 is counterintuitive in the context of the significant elevation of E2F target gene expression in Cry $2^{-1-}$ MEFs. However, we only assessed the impact of CRY regulation of a select subset of the E2F family; it is possible that E2F2 and/or E2F3 are significantly stabilized in the absence of CRY2 and contribute to the observed effects on gene expression. Conversely, other E2F repressors may be more responsive to the genetic manipulation of Crys than E2F4 and E2F8 are. Given that CRYs repress the transcriptional activity of CLOCK-BMAL1 ${ }^{28}$ and of several nuclear hormone receptors ${ }^{9}$, CRYs may suppress the transcriptional activity of activator E2Fs or directly mediate transcriptional repression conferred by repressor E2Fs. If they do, that could also explain the increased expression 
A

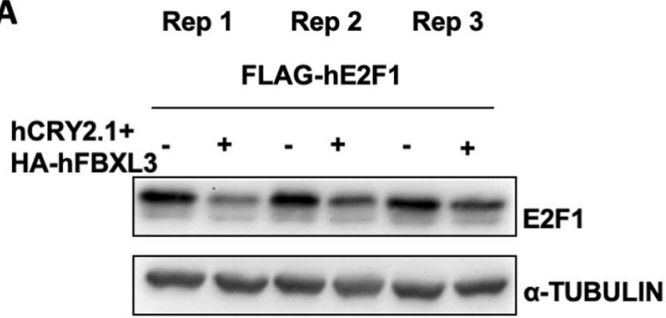

B

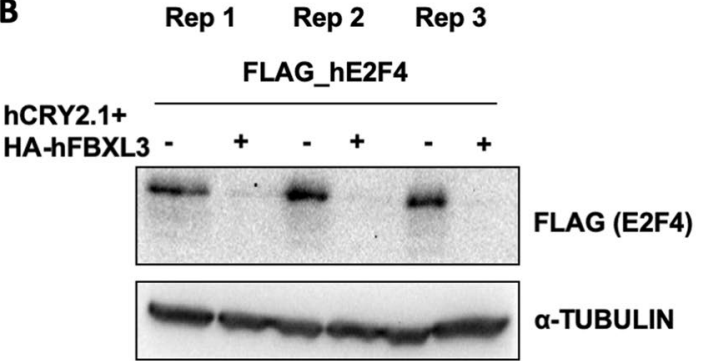

C

$\operatorname{Rep} 1 \quad \operatorname{Rep} 2 \quad \operatorname{Rep} 3$ FLAG-hE2F8

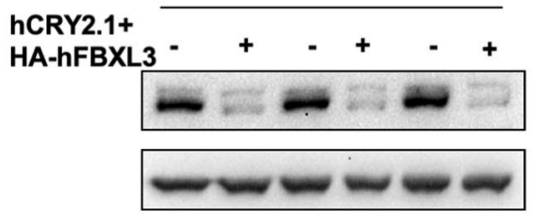

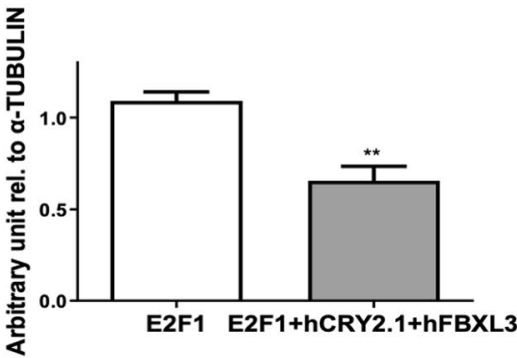

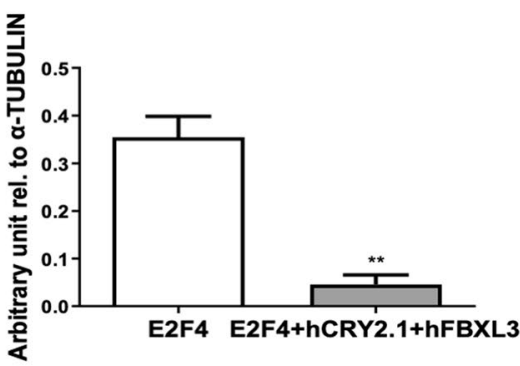

LAG (E2F8) $\alpha-T U B U L I N$

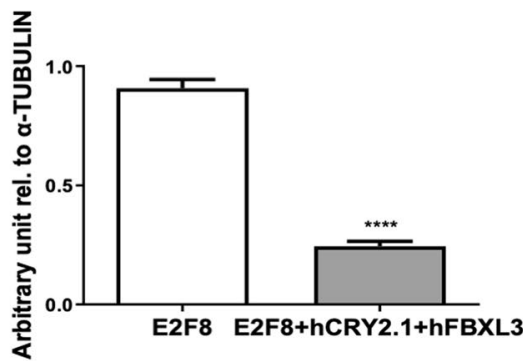

Figure 3. E2F steady state protein levels are affected by CRY2 and FBXL3. (A-C) Steady state protein levels of FLAG-E2F1, E2F4, and E2F8 in the indicated conditions detected by IB (-/+ hCRY2.1 and HA-hFBXL3). Data in bar graphs indicate mean \pm s.e.m. of biological triplicates (Replicate (Rep) 1-3) normalized to $\alpha$-TUBULIN. $* * \mathrm{p}<0.01, * * * * \mathrm{p}<0.0001$ using $t$-test.

of E2F target genes in Cry2 $2^{-1-}$ cells. Indeed, analyzing Chromatin-immunoprecipitation (ChIP) sequencing data from mouse livers across circadian time (GSE39860) ${ }^{8}$ reveals that endogenous CRY 1 and CRY2 are associated with the genomic loci containing many of the 200 "hallmark" E2F target genes in mouse livers, and at least some of these sites are not bound by other circadian transcription factors, supporting the idea that CRYs could independently regulate E2F family members bound to chromatin (Table S2). Additionally, E2Fs could have unexpected transcriptional activities in the context of CRY-deficient cells. Finally, some evidence suggests that E2F family members cannot be easily classified into activators and repressors ${ }^{29}$, and the increased expression of E2F target genes in $\mathrm{Cr} y 2^{-1-}$ AMEFs may reflect increased target gene activation by E2F family members that are thought to act as repressors.

A recent study demonstrated that CYCLIN F interacts with and promotes the degradation of activator E2Fs to restrict E2F activity to the S phase of the cell cycle ${ }^{24}$. Our finding that CRYs modulate E2F protein abundance suggests a mechanism by which their abundance and activity could be regulated by circadian cycles. Disruption of circadian rhythms, such as that experienced by shift workers, increases the risk of cancer and other pathologies ${ }^{30}$. E2Fs have been implicated in cancer ${ }^{18,30-32}$, and disruption of their modulation by CRYs could contribute to the elevated cancer risk caused by circadian disruption.

\section{Methods}

Cell culture. Cell culture methods were the same as in ${ }^{17}$. In brief, all primary mouse embryonic (MEF) and adult mouse ear (AMEF) fibroblasts cells were prepared from embryos collected at E15.5 or from ear biopsies of adult mice respectively, and were passaged no more than 10 times as in ${ }^{17}$. Cells were grown in complete Dulbecco's Modified Eagle Medium (DMEM) (Invitrogen cat \#10569) supplemented with 10\% fetal bovine serum (FBS) (HEK 293T cells) or 15\% FBS (MEFs and AMEFs), and 1\% penicillin and streptomycin. HEK 293T cells were grown in a $37^{\circ} \mathrm{C}$ incubator maintained at $5 \% \mathrm{CO}_{2}$ and $20 \% \mathrm{O}_{2}$ (high oxygen) and $\mathrm{MEF}$ cells were grown in a $37^{\circ} \mathrm{C}$ incubator maintained at $5 \% \mathrm{CO}_{2}$ and $3 \% \mathrm{O}_{2}$ (low oxygen). Transfections were carried out using polyethylenimine (PEI; Polysciences Inc catalog \#23966-2) by standard protocols. After 24 hours, the HEK 293T cell media was again replaced and protein extracts were isolated 48 hours post transfection. Cycloheximide (CHX) (Fisher 

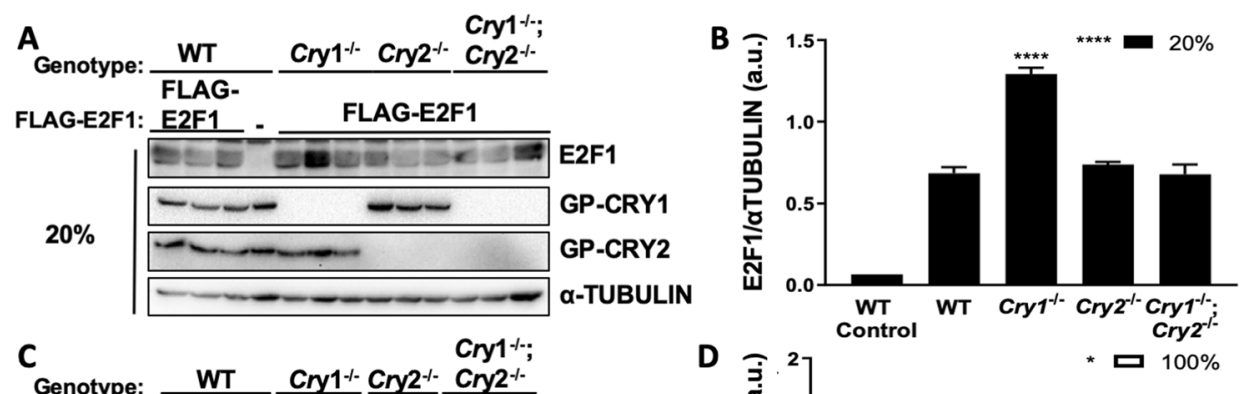

Genotype: WT Cry 1- ${ }^{-1}$ ry2 ${ }^{-1-} \mathrm{Cry2}^{-1-}$

FLAG-E2F1: - FLAG-E2F1

$100 \%$

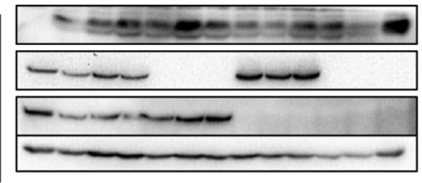

Cry1

E

Genotype: WT $\mathrm{Cry1}^{-1-} \mathrm{Cry2}^{-1-} \mathrm{Cry2}^{-1-}$

FLAG-E2F4: - FLAG-E2F4

$20 \%$

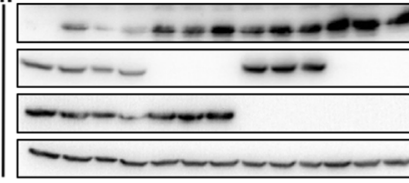

FLAG

GP-CRY1

GP-CRY2

$\alpha$-TUBULIN

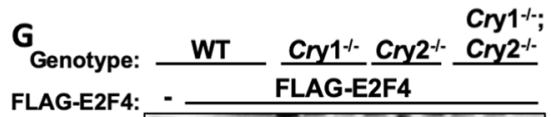

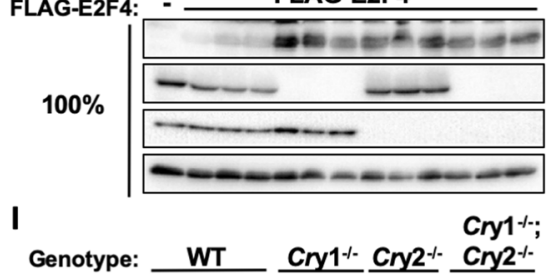

FLAG-E2F8: - FLAG-E2F8

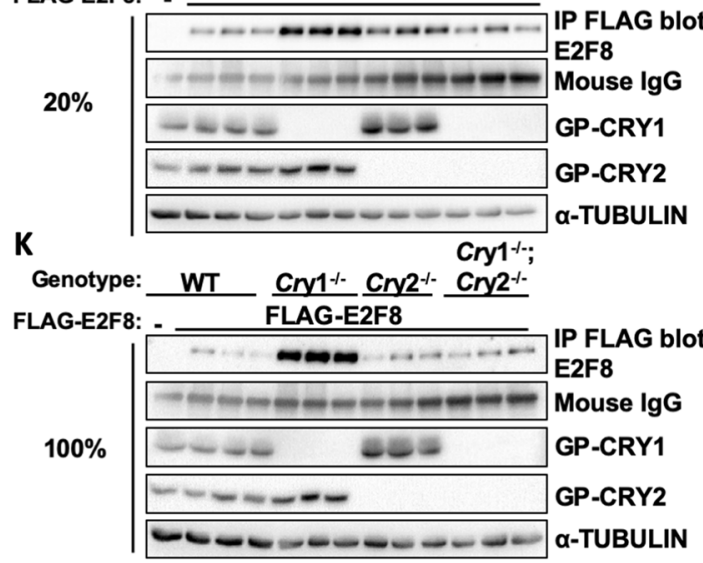

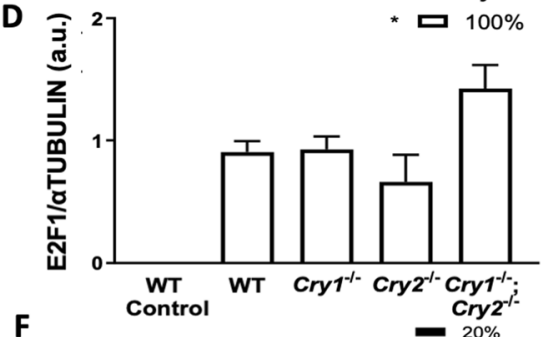

F

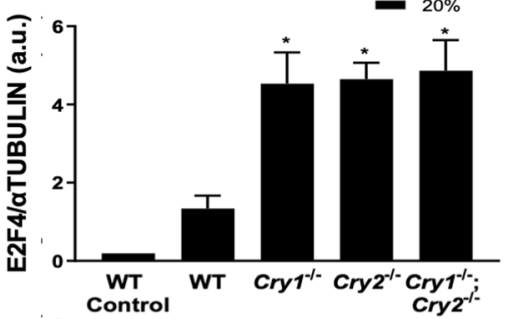

H
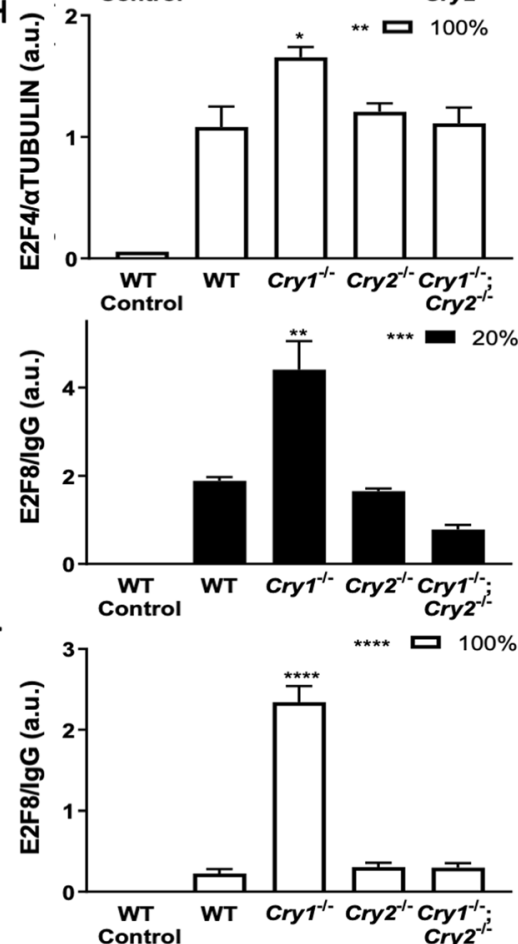

Figure 4. Endogenous CRY1 and CRY2 affect E2F1, E2F4, and E2F8 protein abundance. (A,C,E,G,I,K) Proteins detected by IB in WCL or following FLAG IP (I,K) from AMEFs of the indicated genotypes stably overexpressing tetracycline-inducible FLAG-tagged human E2F1 (A,C), E2F4 (E,G), or E2F8 (I,K) and treated with $1 \mu \mathrm{M}$ doxycycline or vehicle $(-)$. For $(\mathbf{I}, \mathbf{K})$, E2F8 protein levels were detected following FLAG IP. (B,D,F,H,J,L) Quantification of data shown in (A,C,E,G,I,K). Data represent the mean \pm s.e.m. of three biological replicates for cells plated at $20 \%$ (black) or $100 \%$ (white) confluency. $* \mathrm{p}<0.05, * * \mathrm{p}<0.01$, $* * * \mathrm{p}<0.001, * * * * \mathrm{p}<0.0001$ by one-way ANOVA with Dunnett's multiple comparisons: P values for a main effect of genotype are listed next to legend; $\mathrm{P}$ values shown above bars represent post-hoc comparison to WT). 
cat\# 50255724) was used at a concentration of $100 \mu \mathrm{g} / \mathrm{mL}$ as indicated. Cells were treated with $10 \mu \mathrm{M}$ MG-132 (Sigma cat\# C2211-5MG) for 4-5 hrs prior to lysis.

Generation of viruses and stable cell lines. Methods were the same $\mathrm{as} \mathrm{in}^{17}$ except lentiviruses expressing pCW-2xFLAG-E2F1 E2F4, or E2F8 were used.

Doxycycline induction of stable cell lines. Methods were the same as in ${ }^{17}$ : AMEF cells of the indicated genotypes stably expressing pCW-2xFLAG-E2F1, pCW-2xFLAG-E2F4, or pCW-2xFLAG-E2F8 were treated with $1 \mu \mathrm{M}$ doxycycline (Sigma cat \# D9891) for 48 hours to induce 2xFLAG-E2F1 or 2xFLAG-E2F4 expression.

Immunoprecipitation and western blotting. HEK 293T whole cell extracts for co-IP were prepared using lysis buffer containing 1\%TX-100, $50 \mathrm{mM}$ HEPES (pH 7.4), $138 \mathrm{mM} \mathrm{KCl}, 4 \mathrm{mM} \mathrm{NaCl}, 50 \mathrm{mM}$ sodium pyrophosphate, $100 \mathrm{mM}$ sodium fluoride, $10 \mathrm{mM}$ EDTA, $1 \mathrm{mM}$ EGTA pH 8, $50 \mu \mathrm{M}$ PMSF, $1 \mathrm{mM}$ $\beta$-Glycerophosphate, $1 \mathrm{mM}$ sodium orthovanadate, and protease inhibitors (Thermo Scientific cat \# 88265) as previously described (Lamia et al., 2004). Immunoprecipitation was performed using anti-Flag M2 agarose beads (Sigma cat \#A2220). Antibodies for Western Blots were anti-FLAG polyclonal (Sigma cat \#F7425), anti-V5 polyclonal (Bethyl Labs cat\# A190-120A), anti-HA polyclonal (Sigma cat \# H6908), anti- - -TUBULIN (Sigma cat \# T5168), anti-E2F1 (Santa Cruz KH95), anti-E2F8 (Abcam ab109596), CRY1-CT and CRY2-CT ${ }^{11}$. For CHX assay, HEK293T cells were lysed with a RIPA Buffer containing 1\% TX-100, $147 \mathrm{mM} \mathrm{NaCl}, 12 \mathrm{mM}$ Sodium deoxycholate, $0.1 \%$ SDS, $50 \mathrm{mM}$ Tris pH 8.0, $10 \mathrm{mM}$ EDTA, $50 \mu \mathrm{M}$ PMSF, $1 \mathrm{mM} \beta$-Glycerophosphate, $1 \mathrm{mM}$ Sodium Orthovanadate and protease inhibitors (Thermo Scientific cat \# 88265).

Plasmids. pcDNA3-HA-mCRY2, pcDNA3-HA-mCRY1, and pcDNA3-FBXL3-V5 are as previously described $^{13}$. cDNA encoding human E2F1, E2F4, E2F8, $h C R Y 2.1$, and FBXL3 were generated by RT-PCR from RNA extracted from HEK 293T cells. E2F1, E2F4, E2F8, hCRY2.1, and FBXL3 cDNAs were cloned into pcDNA3.1-based FLAG-epitope tagged vector or pcDNA3.1-based HA-epitope tagged vector using standard protocols. The FLAG-epitope tag was removed from pcDNA3.1-based FLAG-epitope tagged hCRY2.1 using Q5 Site-Directed Mutagenesis (New England Biolabs Inc. cat \# E0554S). psPAX plasmid (Addgene plasmid 12260) and pMD2.G plasmid (Addgene plasmid 12259) deposited by Dr. Didier Trono, and used for infection, were purchased from Addgene. pCW-Cas9 was a gift from Eric Lander \& David Sabatini (Addgene plasmid \# 50661) ${ }^{33}$ and the Cas 9 sequence was replaced with 2x-FLAG-E2F1, E2F4, or E2F8 cDNA using the Gibson Assembly Ultra Kit (Synthetic Genomics Inc. cat \# GA1200-10).

Quantitative RT-PCR (qPCR). Methods were the same as in ${ }^{17}$.

Primers used for qPCR

\begin{tabular}{|c|c|c|}
\hline primer name & Forward $\left(5^{\prime}-3^{\prime}\right)$ & Reverse $\left(3^{\prime}-5^{\prime}\right)$ \\
\hline$m-E 2 f 1$ & TGCAGAAACGGCGCATCTAT & CCGCTTACCAATCCCCACC \\
\hline$h-E 2 F 1$ & GAGAACAGGGCCACTGACTCTGCC & GCCGGAGAAGTCCTCCCGCAC \\
\hline$h-E 2 F 4$ & CCCATATGGCGGAGGCCGGGCC & CGCCGCTTCTGGCGTACAGCTAGGG \\
\hline$m-E 2 f 4$ & GGAGCTGCAGCAACGAGAGC & CTAGACTGGTGCCCGATGGC \\
\hline$m-U 36 b 4$ & AGATGCAGCAGATCCGCA & GTTCTTGCCCATCAGCACC \\
\hline$m-M c m 4$ & GAGGAAAGCAGGTCGTCACC & AGGGCTGGAAAACAAGGCATT \\
\hline$m-M c m 6$ & CCTGTGAATAGGTTCAACGGC & CATTTTCCTGAGGTGGAGCAC \\
\hline$m$-Ccne1 & AGCGAGGATAGCAGTCAGCC & GGTGGTCTGATTTTCCGAGG \\
\hline$m-C d k n 1 b$ & ACCCGCCCGAGGAGGAAGAT & CTCGCTTCTTCCATATCCCG \\
\hline$m$-Paics & CAGTTGTTACAGGAAGCTGG & CGTCCTTGAAGAACATCTCC \\
\hline$m-L m n b 1$ & GCTGCTGCTCAATTATGCCAAG & GATGCTTCTAGCTGGGCAATC \\
\hline$m$-Ranbp1 & GATGCGTGCAAAGCTGTTCC & GGTGTAATATAGTGGTTGGCGC \\
\hline$m-E 2 f 2$ & GTGACCTACCAGGATATCCGTG & GCC TTG ACC GCA ATC ACT GTC \\
\hline$m-E 2 f 3 a$ & GCCTCTACACCACGCCACAAG & TCGCCCAGTTCCAGCCTTC \\
\hline$m-E 2 f 3 b$ & CGGAAATGCCCTTACAGC & CTCAGTCACTTCTTTGGACAG \\
\hline$m-E 2 f 4$ & GGAGCTGCAGCAACGAGAGC & CTAGACTGGTGCCCGATGGC \\
\hline$m-E 2 f 5$ & GTGATGGAAGACTCCATTAATAAC & GGCCCTGAGTGACTCTTC \\
\hline$m-E 2 f 6$ & GGCATCGAACTGGTGGAAAAG & CCAACAGTTGCTGAGCACAATC \\
\hline$m-E 2 f 7$ & GAAGTCTGGCGGCCATCTAC & GACCATTCTGCGCAGAGAAGG \\
\hline$m-E 2 f 8$ & CCCTGTCAAGAGCAACAAAGC & CTG TAG GGT CCA GGG GAG \\
\hline$m-M y c$ & GCGACTCTGAAGAAGAGCAAG & GCCTCGGGATGGAGATGAG \\
\hline$m$-Bmal1 & TCAAGACGACATAGGACACCT & GGACATTGGCTAAAACAACAGTG \\
\hline$m-N r 1 d 1$ (RevErba) & CGTTCGCATCAATCGCAACC & GATGTGGAGTAGGTGAGGTC \\
\hline$m-E 2 f 1$ & AGGGAAAGGTGTGAAATCTCC & TTGGTGATGACATAGATGCGC \\
\hline$m-\mathrm{Cr} y 2^{-1-}$ & CCTGGATGCCGATTTCAGTG & ATCGAGAGGGGAAGCCTTTC \\
\hline
\end{tabular}


Gene Set Enrichment Analysis (GSEA). GSEA is a computational method ${ }^{34}$ that determines whether an a priori defined set of genes shows statistically significant, concordant differences between two biological states (e.g., phenotypes). RNA sequencing outputs for WT and Cry $2^{-1-}$ cells were used as input for GSEA comparing the two genotypes (WT versus Cry $2^{-1-}$ regardless of circadian time).

Mice. $C r y 1^{-1-} ; \mathrm{Cr}_{2} 2^{-1-}$ mice from which primary cells were derived were from Dr. Aziz Sancar ${ }^{43}$. All animal care and treatments were in accordance with Scripps Research guidelines and regulations for the care and use of animals. All procedures involving experimental animals were approved by the Scripps Research Institutional Animal Care and Use Committee (IACUC) under protocol \#10-0019.

Accession numbers. The accession number for the RNA sequencing data analyzed in this paper is GEO: GSE89018.

Statistical analysis. Statistics for Fig. 1A were calculated in the Gene Set Enrichment Analysis program as described $^{34}$. Graph Pad Prism 7 was used to compute all other statistics. For Figs. 1C, S1-3 two-way ANOVA with Tukey's multiple comparisons was used. For Fig. 4B,D,F,H,J,L one-way ANOVA with Dunnett's multiple comparisons was used. For Fig. 3, $t$-test was used.

Received: 17 October 2019; Accepted: 12 February 2020;

Published online: 05 March 2020

\section{References}

1. Partch, C. L., Green, C. B. \& Takahashi, J. S. Molecular architecture of the mammalian circadian clock. Trends Cell Biol. 24, 90-99, https://doi.org/10.1016/j.tcb.2013.07.002 (2014).

2. Hunt, T. \& Sassone-Corsi, P. Riding tandem: circadian clocks and the cell cycle. Cell. 129, 461-464, https://doi.org/10.1016/j. cell.2007.04.015 (2007).

3. Matsuo, T. et al. Control mechanism of the circadian clock for timing of cell division in vivo. Sci. 302, 255-259, https://doi. org/10.1126/science.1086271 (2003).

4. Fu, L. \& Lee, C. C. The circadian clock: pacemaker and tumour suppressor. Nat. Rev. Cancer 3, 350-361, https://doi.org/10.1038/ nrc1072 (2003).

5. Nagoshi, E. et al. Circadian gene expression in individual fibroblasts: cell-autonomous and self-sustained oscillators pass time to daughter cells. Cell 119, 693-705, https://doi.org/10.1016/j.cell.2004.11.015 (2004).

6. Kowalska, E. et al. NONO couples the circadian clock to the cell cycle. Proc. Natl Acad. Sci. USA 110, 1592-1599, https://doi. org/10.1073/pnas.1213317110 (2013).

7. Bass, J. Circadian topology of metabolism. Nat. 491, 348-356, https://doi.org/10.1038/nature11704 (2012).

8. Koike, N. et al. Transcriptional architecture and chromatin landscape of the core circadian clock in mammals. Sci. 338, 349-354, https://doi.org/10.1126/science.1226339 (2012).

9. Kriebs, A. et al. Circadian repressors CRY1 and CRY2 broadly interact with nuclear receptors and modulate transcriptional activity. Proc. Natl Acad. Sci. USA 114, 8776-8781, https://doi.org/10.1073/pnas.1704955114 (2017).

10. Jordan, S. D. et al. CRY1/2 Selectively Repress PPARdelta and Limit Exercise Capacity. Cell. Metab. 26, 243-255 e246, https://doi. org/10.1016/j.cmet.2017.06.002 (2017).

11. Lamia, K. A. et al. Cryptochromes mediate rhythmic repression of the glucocorticoid receptor. Nat. 480, 552-556, https://doi. org/10.1038/nature10700 (2011).

12. Qu, M., Duffy, T., Hirota, T. \& Kay, S. A. Nuclear receptor HNF4A transrepresses CLOCK:BMAL1 and modulates tissue-specific circadian networks. Proc. Natl. Acad. Sci. USA 115, E12305-E12312, https://doi.org/10.1073/pnas.1816411115 (2018).

13. Huber, A. L. et al. CRY2 and FBXL3 Cooperatively Degrade c-MYC. Mol. Cell. 64, 774-789, https://doi.org/10.1016/j. molcel.2016.10.012 (2016).

14. Sakamoto, W. \& Takenoshita, S. Overexpression of Both Clock and Bmal1 Inhibits Entry to S Phase in Human Colon Cancer Cells. Fukushima J. Med. Sci. 61, 111-124, https://doi.org/10.5387/fms.2015-11 (2015).

15. Gotoh, T. et al. The circadian factor Period 2 modulates p53 stability and transcriptional activity in unstressed cells. Mol. Biol. Cell. 25, 3081-3093, https://doi.org/10.1091/mbc.E14-05-0993 (2014).

16. Gotoh, T. et al. Model-driven experimental approach reveals the complex regulatory distribution of p53 by the circadian factor Period 2. Proc. Natl Acad. Sci. USA 113, 13516-13521, https://doi.org/10.1073/pnas.1607984113 (2016).

17. Correia, S. P. et al. The circadian E3 ligase complex SCF(FBXL3+CRY) targets TLK2. Sci. Rep. 9, 198, https://doi.org/10.1038/ s41598-018-36618-3 (2019).

18. Attwooll, C., Lazzerini Denchi, E. \& Helin, K. The E2F family: specific functions and overlapping interests. EMBO J. 23, 4709-4716, https://doi.org/10.1038/sj.emboj.7600481 (2004).

19. Blanchet, E. et al. E2F transcription factor-1 regulates oxidative metabolism. Nat. Cell. Biol. 13, 1146-1152, https://doi.org/10.1038/ ncb2309 (2011).

20. Marti, A., Wirbelauer, C., Scheffner, M. \& Krek, W. Interaction between ubiquitin-protein ligase SCFSKP2 and E2F-1 underlies the regulation of E2F-1 degradation. Nat. Cell. Biol. 1, 14-19, https://doi.org/10.1038/8984 (1999).

21. Budhavarapu, V. N. et al. Regulation of E2F1 by APC/C Cdh1 via K11 linkage-specific ubiquitin chain formation. Cell. Cycle 11, 2030-2038, https://doi.org/10.4161/cc.20643 (2012).

22. Cohen, M. et al. Unbiased transcriptome signature of in vivo cell proliferation reveals pro- and antiproliferative gene networks. Cell. Cycle 12, 2992-3000, https://doi.org/10.4161/cc.26030 (2013).

23. Ping, Z., Lim, R., Bashir, T., Pagano, M. \& Guardavaccaro, D. APC/C (Cdh1) controls the proteasome-mediated degradation of E2F3 during cell cycle exit. Cell. Cycle 11, 1999-2005, https://doi.org/10.4161/cc.20402 (2012).

24. Clijsters, L. et al. Cyclin F Controls Cell-Cycle Transcriptional Outputs by Directing the Degradation of the Three Activator E2Fs. Mol. Cell. 74, 1264-1277 e1267, https://doi.org/10.1016/j.molcel.2019.04.010 (2019).

25. Subramanian, A. et al. Gene set enrichment analysis: a knowledge-based approach for interpreting genome-wide expression profiles. Proc. Natl Acad. Sci. U S A. 102, 15545-15550, https://doi.org/10.1073/pnas.0506580102 (2005).

26. Liberzon, A. et al. The Molecular Signatures Database (MSigDB) hallmark gene set collection. Cell. Syst. 1, 417-425, https://doi. org/10.1016/j.cels.2015.12.004 (2015).

27. Gotoh, T., Vila-Caballer, M., Liu, J., Schiffhauer, S. \& Finkielstein, C. V. Association of the circadian factor Period 2 to p53 influences p53's function in DNA-damage signaling. Mol. Biol. Cell. 26, 359-372, https://doi.org/10.1091/mbc.E14-05-0994 (2015).

28. Griffin, E. A. Jr., Staknis, D. \& Weitz, C. J. Light-independent role of CRY1 and CRY2 in the mammalian circadian clock. Sci. 286, 768-771, https://doi.org/10.1126/science.286.5440.768 (1999). 
29. Wu, L. et al. The E2F1-3 transcription factors are essential for cellular proliferation. Nat. 414, 457-462, https://doi. org/10.1038/35106593 (2001).

30. Kelleher, F. C., Rao, A. \& Maguire, A. Circadian molecular clocks and cancer. Cancer Lett. 342, 9-18, https://doi.org/10.1016/j. canlet.2013.09.040 (2014).

31. Kent, L. N. \& Leone, G. The broken cycle: E2F dysfunction in cancer. Nat. Rev. Cancer 19, 326-338, https://doi.org/10.1038/s41568019-0143-7 (2019).

32. Chen, H. Z., Tsai, S. Y. \& Leone, G. Emerging roles of E2Fs in cancer: an exit from cell cycle control. Nat. Rev. Cancer 9, 785-797, https://doi.org/10.1038/nrc2696 (2009).

33. Wang, T., Wei, J. J., Sabatini, D. M. \& Lander, E. S. Genetic screens in human cells using the CRISPR-Cas9 system. Sci. 343, 80-84, https://doi.org/10.1126/science.1246981 (2014).

34. Subramanian, A., Kuehn, H., Gould, J., Tamayo, P. \& Mesirov, J. P. GSEA-P: a desktop application for Gene Set Enrichment Analysis. Bioinforma. 23, 3251-3253, https://doi.org/10.1093/bioinformatics/btm369 (2007).

\section{Acknowledgements}

This work was supported by NIH grants CA211187 and DK112927 (to K.A.L.). We thank Drs. Eros LazzeriniDenchi and Stephanie Papp Correia for helpful discussions.

\section{Author contributions}

A.B.C., A.-L.H., and K.A.L. designed research. A.B.C. and A.-L.H. performed experiments. K.A.L. supervised the study, performed GSEA. A.B.C. and K.A.L. wrote the paper. All authors edited and approved the manuscript.

\section{Competing interests}

The authors declare no competing interests.

\section{Additional information}

Supplementary information is available for this paper at https://doi.org/10.1038/s41598-020-61087-y.

Correspondence and requests for materials should be addressed to K.A.L.

Reprints and permissions information is available at www.nature.com/reprints.

Publisher's note Springer Nature remains neutral with regard to jurisdictional claims in published maps and institutional affiliations.

(c) (i) Open Access This article is licensed under a Creative Commons Attribution 4.0 International License, which permits use, sharing, adaptation, distribution and reproduction in any medium or format, as long as you give appropriate credit to the original author(s) and the source, provide a link to the Creative Commons license, and indicate if changes were made. The images or other third party material in this article are included in the article's Creative Commons license, unless indicated otherwise in a credit line to the material. If material is not included in the article's Creative Commons license and your intended use is not permitted by statutory regulation or exceeds the permitted use, you will need to obtain permission directly from the copyright holder. To view a copy of this license, visit http://creativecommons.org/licenses/by/4.0/.

(C) The Author(s) 2020 